\title{
A novel approach in magnetic cloud-driven Forbush decrease modeling
}

\author{
Simone Benella ${ }^{\circledR 1}$, Monica Laurenza ${ }^{\circledR 1}$, Rami Vainio ${ }^{\circledR 2}$, Catia Grimani ${ }^{\circledR 3}$, Giuseppe Consolini ${ }^{\circledR 1}$, \\ Qiang $\mathrm{Hu}^{4}$, Alexandr Afanasiev ${ }^{-5}$

\section{Correspondence} \\ 'INAF-Istituto di Astrofisica e Planetologia Spaziali, Roma, Italy, \\ simone.benella@inaf.it, monica.laurenza@inaf.it, giuseppe.consolini@inaf.it \\ 2Department of Physics and Astronomy, University of Turku, Finland, rami.vainio@utu.fi \\ ${ }^{3}$ DiSPeA, Università degli Studi di Urbino Carlo Bo, Urbino, Italy; Istituto Nazionale di Fisica Nucleare (INFN), \\ Sezione di Firenze, Sesto Fiorentino, Italy, catia.grimani@uniurb.it \\ ${ }^{4}$ Center for Space Plasma and Aeronomic Research, The University of Alabama in Huntsville, Huntsville, USA, \\ qh0001@uah.edu \\ ${ }^{5}$ Department of Physics and Astronomy, University of Turku, Turku, Finland, alexandr.afanasiev@utu.fi
}

\section{OPEN ACCESS}

This work is published under the Creative Commons Attribution 4.0 International license (CC BY 4.0) Please note that individual, appropriately marked parts of the work may be excluded from the license mentioned or may be subject to other copyright conditions. If such third party materiatis not under the Cretive Commons license, under the Creative Commons license, any copying, duction is only permitted with the prior consent of the respective copy right owner or on the basis of relevan legal authorization regulations.

\section{Keywords}

cosmic rays; solar-terrestrial relations; coronal mass ejections (CMEs); magnetic clouds

\begin{abstract}
Interplanetary coronal mass ejections (ICMEs) are large-scale solar wind disturbances propagating from the Sun and causing a depression of the galactic-cosmic ray (GCR) intensity known as Forbush decrease (FD). ICMEs generally contain coherent plasma structures called magnetic clouds (MCs). A unique and powerful data analysis tool allowing for the study of the quasi-3-D configuration of a MC is the Grad-Shafranov (GS) reconstruction. The aim of this work is to investigate the role played by the MC configuration in the formation of a FD. A suited full-orbit test-particle simulation has been developed in order to evaluate FD amplitude and time profile produced by the MC obtained with the GS reconstruction. Particle trajectories are computed starting from an isotropic flux outside the MC region. In addition, particle diffusion has been modeled by superimposing a small-angle scattering over the unperturbed charged particle motion at each time step. The model allows us to investigate the MC effect on GCR propagation and to study the energy dependence of the physical processes involved, as it provides an estimate of ground-based GCR counts observations at different latitudes. A comparison between model results and both space-based cosmic-ray measurements in L1 and ground-based observations suggests a major role of drifts in producing the FD and a reduced contribution of GCR particle diffusion.
\end{abstract}

\section{Introduction}

Interplanetary coronal mass ejections (ICMEs) are large magnetic structures connected to the Sun that during their travel can limit the cosmic-ray propagation producing galactic cosmic-ray (GCR) intensity depressions known as Forbush decreases (FDs; Forbush 1937). From a statistical point of view the $80 \%$ of ICMEs is associated with a FD (Richardson \& Cane 2011). During the ICME transit it is generally possible to identify two components: the first comprises a shock front and a region of shocked and compressed solar wind plasma presenting large-amplitude magnetic field fluctuations called turbulent sheath; the second is a coherent plasma structure called magnetic cloud (MC) that is presumably connected to the Sun (see Cane 2000, and references therein). The 
typical signatures of a $\mathrm{MC}$ on the in situ spacecraft $(\mathrm{S} / \mathrm{C})$ observations are a smooth rotation in the magnetic field components, a magnetic field strength higher than the background solar wind and values of temperature and plasma-beta that are lower than average (Burlaga et al. 1990; Lepping et al. 1990, 1997). In general, the most effective physical process in generating major FDs is the interaction of GCRs with the ICME shock/sheath region, since intense shocks associated with fast ICMEs may overcome the MC effect in GCR modulation (e.g. Wibberenz et al. 1998). Otherwise, the $\mathrm{MC}$ effect could even be dominant since the minimum intensity occurs after the arrival of the MC (Badruddin 1986; Zhang \& Burlaga 1988). Moreover, ICMEs that contain MCs cause larger FDs on average with respect to ICMEs that do not show any coherent structure (Richardson \& Cane 2011). In this work we focus on slow ICMEs for which the effect of the shock/sheath and the effect of the MC on the GCR intensity could be similar. Therefore, in case of weak shock/sheath part of an ICME the FD profile depends on the magnetic configuration within the ICME. Numerous models have been developed in order to relate the properties of FDs with those of ICMEs/MCs (Cane et al. 1995; Munakata et al. 2006; Krittinatham \& Ruffolo 2009; Dumbović et al. 2018; Petukhova et al. 2019). These studies are based on the analytical definition of the magnetic field components within the $\mathrm{MC}$ and in some cases the MC expansion is taken into account into the model. In this work we propose an novel method to model MC-driven FDs by using a realistic MC configuration starting from IMF and plasma in situ data. The quasi-3-D large-scale configuration of a MC can be found by applying the Grad-Shafranov (GS) reconstruction (Hau \& Sonnerup 1999; Hu \& Sonnerup 2002). The main idea of the method is to join the GS reconstruction with a suited test-particle simulation that allows us to investigate the effect of the MC configuration in modulating the GCR intensity. Finally, we present a case study for which the method can be applied successfully. In order to test the model we use ground-based GCR observations from five neutron monitor (NM) stations at different latitudes (Dome C, South Pole, Newark, Rome and Mexico City) as well as space-based measurements gathered by a radiation monitor on board the LISA Pathfinder mission (LPF; Armano et al. 2018, 2019). Space-based GCR observations in the near-Earth environment reach lower rigidities with respect to NMs and allow for the study of the fine structure of FD evolution. On the other hand, a comparative study with ground-based NM observations is crucial in order to investigate the energy dependence of this event. The paper is organized as follows. In Section 2 a description of the method is provided and a comparison between model results and observations is reported in Section 3 as a case study. Finally, our conclusions are drawn.

\section{Description of the method}

The method that we present is aimed to simulate the GCR variation profile of a FD event associated with the passage of a MC. In order to investigate the effect of the MC in modulating GCR particles a quasi-3-D magnetic field configuration representing the MC is necessary. The magnetic structure is obtained here by applying the GS reconstruction and it is used to initialize the particle simulation. The GS reconstruction is an advanced data analysis technique that allows to retrieve a MC topology starting from single $\mathrm{S} / \mathrm{C}$ in situ data. The technique is based on the GS equation, that describes a $2 \mathrm{D}$ plasma structure under the hypothesis of axial invariance along the $z$-axis:

$$
\frac{\partial^{2} A}{\partial x^{2}}+\frac{\partial^{2} A}{\partial y^{2}}=-\mu_{0} \frac{d}{d A}\left(p+\frac{B_{Z}^{2}}{2 \mu_{0}}\right),
$$

where $A$ is the vector potential, $p$ is the plasma pressure and $\mu_{0}$ is the vacuum permeability. The first step in performing the GS reconstruction is the application of the deHoffmann-Teller (HT) analysis. This procedure is aimed to estimate the HT velocity that represents the mean velocity of the MC, used to fix the comoving reconstruction reference frame. In this reference frame the 
electric field vanishes and the magnetic structure is stationary, according to the Faraday's law. The zaxis orientation of the reconstruction reference frame can be estimated by using the condition that the transverse pressure

$$
P_{t}=p+\frac{B_{z}^{2}}{2 \mu_{0}}
$$

is a single-valued function of the potential vector $A$. The $\mathrm{x}$-axis is oriented along the $\mathrm{S} / \mathrm{C}$ trajectory direction, where the magnetic vector potential $A(x, 0)$ is calculated from data. The transverse pressure is then fitted with a proper combination of analytical functions and equation (1) is numerically evaluated. The GCR intensity modulation associated with the MC transit is modeled through a full-orbit test-particle simulation (Benella 2020; Benella et al. 2020). Particles injected in the simulation are only protons, that represent the $90 \%$ of the GCR composition in the inner heliosphere, approximately. The incident proton flux is assumed to be isotropic. The hypothesis of isotropy holds since we focused on slow ICMEs with no clear shock/sheath effect on GCRs. Otherwise, when the interaction between shock/sheath region and GCRs is important, the particle flux may be anisotropic at the MC start time (Tortermpun et al. 2018). The aim of this test-particle simulation is to provide a proton intensity variation profile as a function of time. First, the particle motion through the steady magnetic field is determined by drifts induced by magnetic field inhomogeneity and curvature, thus leading to fully deterministic particle trajectories, since random fluctuations of the magnetic field components are not allowed. As a second step, we evaluated the contribution of the particle diffusion in affecting the proton propagation in the simple assumption of constant diffusion coefficient $D$. The particle diffusion is modeled by superimposing a small-angle scattering over the unperturbed charged particle motion at each time step $\Delta t$. The scattering process varies the particle velocity vector direction with an angle $\theta_{s c}$ at each time step and preserves the particle energy. The square of the scattering angle $\theta_{s c}$ is exponentially distributed. The mean-free path of the diffusion process is defined as $\lambda=3 D / v$, where $v$ is the particle velocity. The proton intensity variation profile obtained with and without the diffusion process are compared and discussed in the next section.

\section{The 2016 August 2nd Forbush decrease}

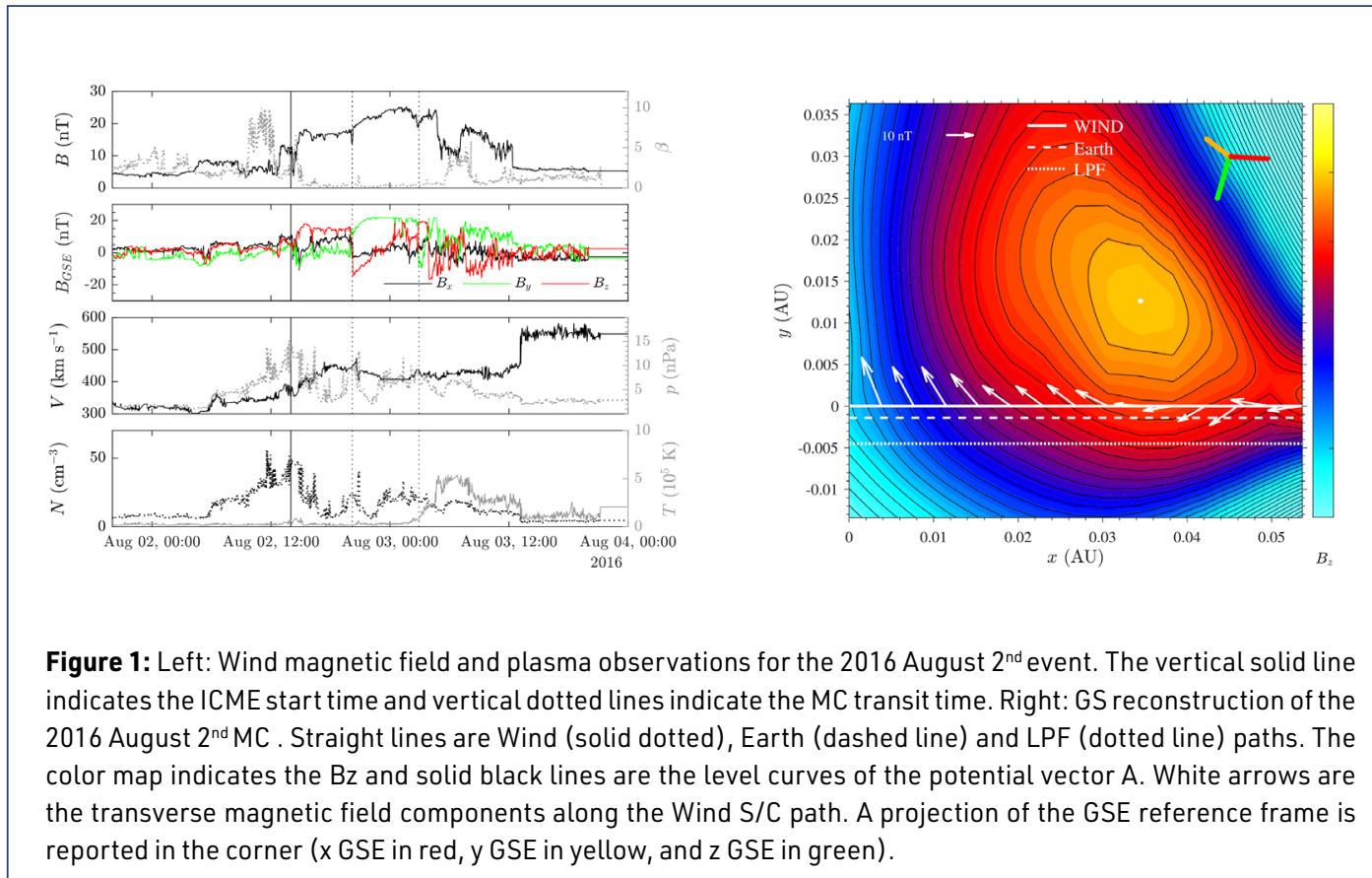




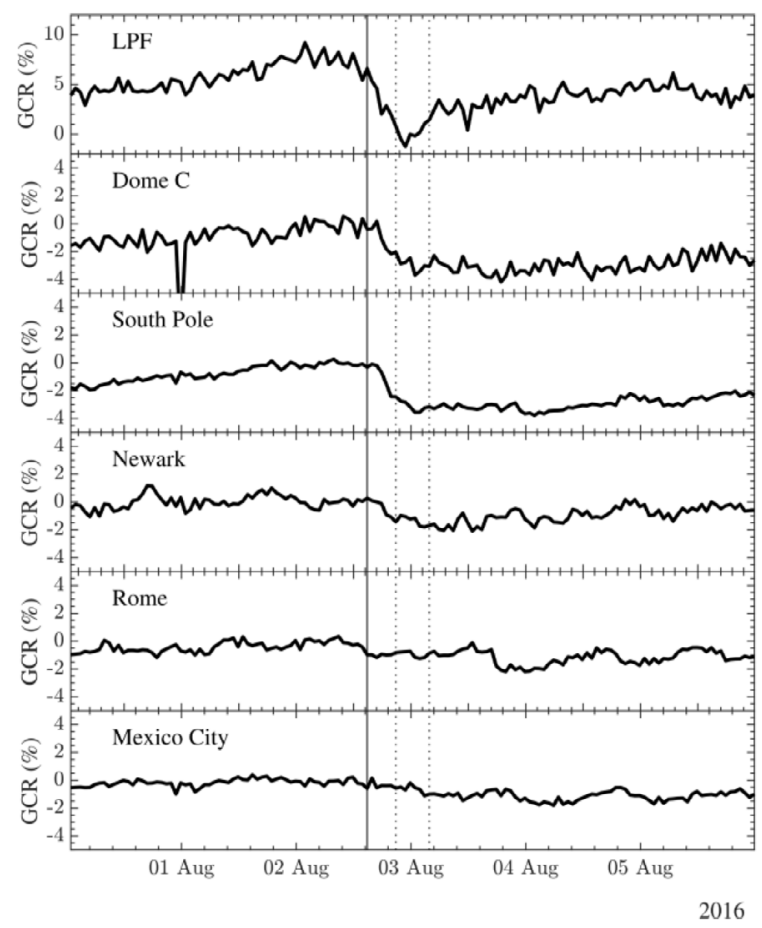

Figure 2: LPF (first panel) and NM (other panels) GCR observations for the 2016 August $2^{\text {nd }} \mathrm{FD}$. The vertical solid line indicates the ICME start time and vertical dotted lines indicate the MC transit time.

An ICME was observed at L1 between 2016 August $2^{\text {nd }}$ at 14:00 UT and August $3^{\text {rd }}$ at 03:00 UT, as reported in the near-Earth ICME catalog at http://www.srl.caltech.edu/ACE/ASC/DATA/level3/ icmetable2.html (last accessed April 7, 2021). In order to perform the GS reconstruction we used data from the Wind $\mathrm{S} / \mathrm{C}$ that are shown in figure 1 (left panel). The $\mathrm{MC}$ region can be identified between 20:10 UT on August $2^{\text {nd }}$ and 03:00 UT on August $3^{\text {rd }}$, where the smooth rotation of magnetic field components and low temperature and plasma beta values are present. At the ICME transit a GCR intensity FD was observed in space by a particle detector on board the LPF S/C at L1 and at
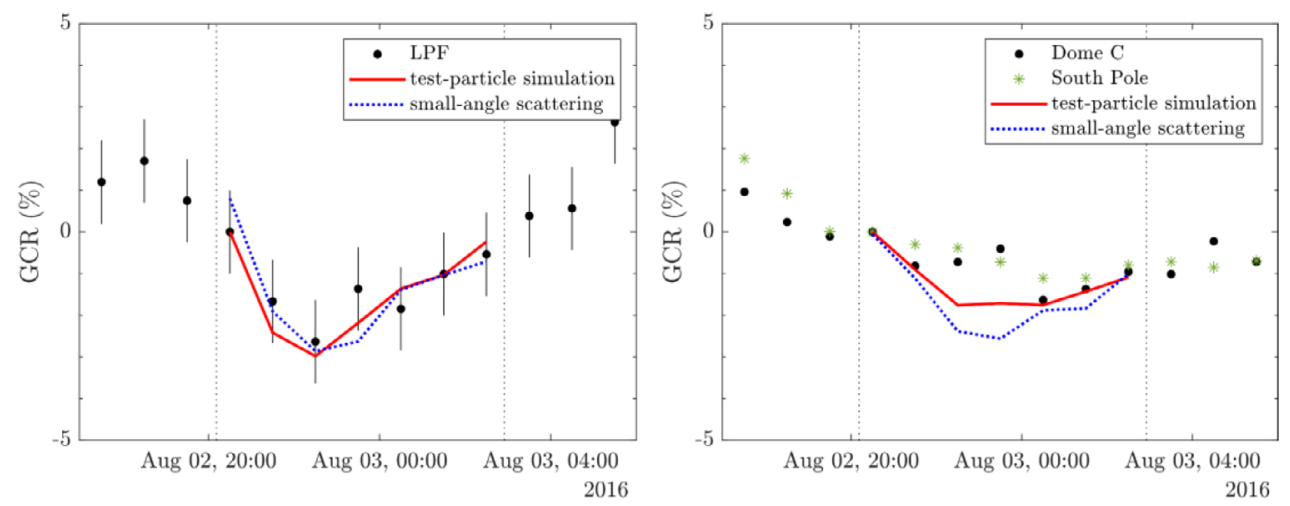

Figure 3: Left: comparison between LPF observations and the hourly-averaged intensity variation profile obtained from the simulation. Right: comparison between polar NM observations (Dome C, South Pole) and the hourly-averaged intensity variation profile obtained from the test-particle simulation. The simulation is performed without including diffusion (red solid lines) and with $D=10^{22} \mathrm{~cm}^{2} \mathrm{~s}^{-1}$ (blue dotted lines). 


\begin{tabular}{|l|l|l|l|l|l|}
\hline & Dome C & South Pole & Newark & Rome & Mexico City \\
\hline Geographic coordinates & $75.06 \mathrm{~S}, 123.2 \mathrm{E}$ & $90.0 \mathrm{~S}, \mathrm{~N} / \mathrm{A}$ & $39.7 \mathrm{~N}, 75.8 \mathrm{~W}$ & $41.9 \mathrm{~N}, 12.5 \mathrm{E}$ & $19.3 \mathrm{~N}, 260.8 \mathrm{E}$ \\
Altitude (m) & 3233 & 2820 & 50 & 2.4 & 2274 \\
Vertical cutoff rigidity (GV) & 1.1 & 1.1 & 1.8 & 6.27 & 8.2 \\
Observed FD amplitude (\%) & 3.7 & 2.6 & $<1$ & 1.1 & $<1$ \\
Observed MC-driven FD ampl. (\%) & 1.7 & 1.2 & 1.1 & 1.0 & $<1$ \\
Simulated MC-driven FD ampl. (\%) & 1.8 & 1.8 & & \\
\hline
\end{tabular}

Table 1: Geographic latitude, altitude, and vertical cutoff rigidities of five NM stations. The total and MC-driven FD amplitudes of the 2016 August 2 event are compared with simulation results for the MC-driven FD amplitude.

ground-level by NMs. LPF and NM GCR observed variations are reported in figure 2. The GS reconstruction is then performed. The HT analysis returns an excellent constant velocity $V_{H T}=(-413.6,-26.4,11.5) \mathrm{km} \mathrm{s}^{-1}$ and a $\mathrm{z}$-axis orientation with respect to the geocentric solar ecliptic (GSE) reference frame is $\vartheta=17.3^{\circ}$ and $\varphi=53.9^{\circ}$, where $\vartheta$ and $\varphi$ are the latitude and longitude angles respectively. The GS reconstruction is shown in figure 1 (right panel). Straight lines at $y=0, \mathrm{y}=-1.4 \times 10^{-3} \mathrm{AU}$ and $\mathrm{y}=-4.4 \times 10^{-3} \mathrm{AU}$ indicate Wind, Earth and LPF paths through the MC, respectively. The last step of our method is the test-particle simulation. The initial proton differential flux is calculated with the Gleeson and Axford model (Gleeson \& Axford 1968) by setting the proton local interstellar spectrum according to Usoskin et al. (2017). Hence, the solar modulation parameter for 2016 August is $\phi=519 \mathrm{MV}$. The energy interval of the protons injected in the simulation is set between $70 \mathrm{MeV}$ and $100 \mathrm{GeV}$ since LPF measured proton and helium nuclei above 70 $\mathrm{MeV} n^{-1}$. In order to estimate the NM count rate from the simulation we cut the proton differential flux above the cutoff energy of each NM station and used the yield function proposed by Mishev et al. (2013) to take into account the instrument response. NM characteristics, observations and MCdriven FD amplitudes obtained from the simulation for these five NM stations are summarized in table 1. The signature of the MC passage on the GCR flux is a decrease of the particle intensity of $1.7 \%$ and $1.2 \%$ at Dome $\mathrm{C}$ and South Pole, respectively. The cutoff energy is the atmospheric cutoff of $E_{c}=500 \mathrm{MeV}$ for these polar stations. The simulation outcome is a decrease of $1.8 \% \pm 0.4 \%$ at Dome $\mathrm{C}$ and South Pole locations. The FD energy dependence is obtained through the simulation as the FD amplitude goes down at higher energies as observed by NMs. Indeed, amplitudes $<1 \%$ were recorded by NM stations such as Newark, $E_{c}=1.6 \mathrm{GeV}$, Rome, $E_{c}=5.4 \mathrm{GeV}$, and Mexico City, $E_{c}=7.3 \mathrm{GeV}$. Proton variation time profiles obtained through the simulation are reported in figure 3 where are compared with data gathered by LPF (left panel) and two polar NMs (right panel). Solid red lines are the simulation outcome without diffusion. In this case the modulation of the proton intensity induced by the MC structure is due to drifts associated with the inhomogeneity and the curvature of the magnetic field. There is a good agreement between model results and observations for both FD amplitude and time profile. In general, the cosmic-ray particle diffusion due to turbulent fluctuations of the magnetic field plays an important role in particle intensity modulation in the heliosphere. On the other hand, spatial scales involved in the GCR diffusion are larger with respect to the scale of the MC under study, thus we expect that diffusion does not play an important role in the 2016 August $2^{\text {nd }}$ FD. We verified this by performing a test-particle simulation, which includes the small-angle scattering for a typical value of the diffusion coefficient usually assumed in the heliosphere: $D=10^{22} \mathrm{~cm}^{2} \mathrm{~s}^{-1}$ (Potgieter 2013). In figure 3 GCR observations are compared with simulation outcomes with particle diffusion (blue dotted lines). As expected the time profile is similar to the one obtained when only drifts are considered, indicating that no effect from diffusion is present, being the associated $\lambda \sim 10^{10} \mathrm{~m}$ greater than the MC size, i.e. $\sim 8 \times 10^{9} \mathrm{~m}$. 


\section{Conclusions}

We proposed a method to study the effect of the interaction between MCs and GCRs by using the GS reconstruction technique that allows us to perform a test-particle simulation on a realistic magnetic field configuration based on in situ S/C observations. The method has been applied to the 2016 August $2^{\text {nd }}$ FD. Magnetic field and plasma observations for the GS reconstruction are taken from the Wind S/C and proton variation profiles obtained from the simulation are compared to data gathered by a particle detector on board LPF and to ground-based observations of five NM stations. Simulation results and data trend show an excellent agreement in both amplitude and time profile of the FD, when particles are assumed to propagate through the MC only by means of gradient and curvature drifts, i.e., when the $\mathrm{MC}$ is treated as a stationary plasma structure and magnetic field fluctuations are not allowed. Moreover, we developed a test-particle simulation with a particle small-angle scattering that allowed us to evaluate the effect of the diffusion in modulating GCRs during the FD. For the typical value $D=10^{22} \mathrm{~cm}^{2} \mathrm{~s}^{-1}$ of the diffusion coefficient, that is assumed to be constant at all energies, the large scale on which the GCR diffusion takes place is greater than the size of the analyzed MC. Thus, we suggest that diffusion does not play a primary role in modulating cosmic-ray particles in this MC case study, which can be can be considered as representative of FDs associated with MCs that are not preceded by a shock/sheath region.

\section{Acknowledgments}

S.B., M.L. and G.C. acknowledge the financial support of the Italian MIUR-PRIN grant 2017APKP7T on Circumterrestrial Environment: Impact of Sun-Earth Interaction. The authors are grateful to the LISA Pathfinder Collaboration for providing the mission particle detector data. The LPF data can be downloaded from https://www.cosmos.esa.int/web/lisa-pathfinder-archive/home. R.V. and A.A. acknowledge the financial support of the Academy of Finland (projects 309939 and 312357). Data from the Wind experiment were obtained from the NASA-CDAWeb website. We acknowledge the NMDB database (https://www.nmdb.eu/) funded under the European Union's FP7 program (contract No. 213007), and the PIs of individual NM stations for providing data.

\section{References}

Armano, M., Audley, H., Baird, J., et al., 2018, ApJ, 854, 113, DOI: https://dx.doi.org/10.3847/1538-4357/aaa774 Armano, M., Audley, H., Baird, J., et al., 2019, ApJ, 874, 167, DOI: https://dx.doi.org/10.3847/1538-4357/ab0c99 Badruddin, R., 1986, SoPh, 105, 413

Benella, S., (2020), Galactic cosmic-ray recurrent and transient short-term variations: advanced data analysis and modeling (Doctoral dissertation, University of Urbino Carlo Bo, Urbino, Italy), http://hdl.handle.net/11576/2673737

Benella, S., Laurenza, M., Vainio, R., et al., 2020, ApJ, 901, 21, DOl: https://dx.doi.org/10.3847/1538-4357/abac59

Burlaga, L., Lepping, R., and Jones, J., 1990, Physics of Magnetic Flux Ropes, Vol. 58, 373, DOI: https://dx.doi.org/10.1029/GM058p0373

Cane, H., Richardson, I., and Wibberenz, G., 1995, ICRC, 4, 377

Cane, H. V., 2000, Cosmic Rays and Earth (Berlin: Springer), 55, DOI: https://dx.doi.org/10.1007/978-94-017-1187-6_4

Dumbović, M., Heber, B., Vršnak, B., Temmer, M., and Kirin, A., 2018, ApJ, 860, 71, DOI: https://dx.doi.org/10.3847/1538-4357/aac2de

Forbush, S. E., 1937, PhRv, 51, 1108, DOI: https://dx.doi.org/10.1103/PhysRev.51.1108.3

Gleeson, L., Axford, W., 1968, ApJ, 154, 1011, DOI: https://dx.doi.org/10.1086/149822

Hau, L.-N., Sonnerup, B. U., 1999, JGRA, 104, 6899, DOI: https://dx.doi.org/10.1029/1999JA900002

Hu, Q., Sonnerup, B. U., 2002, JGRA, 107, SSH, DOI: https://dx.doi.org/10.1029/2001JA000293

Krittinatham, W., Ruffolo, D., 2009, ApJ, 704, 831, DOI: https://dx.doi.org/10.1088/0004-637X/704/1/831

Lepping, R., Burlaga, L., Szabo, A., et al., 1997, JGRA, 102, 14049, DOI: https://dx.doi.org/10.1029/97JA00272

Lepping, R., Jones, J., Burlaga, L., 1990, JGRA, 95, 11957, DOI: https://dx.doi.org/10.1029/JA095iA08p11957

Mishev, A., Usoskin, I., Kovaltsov, G., 2013, JGRA, 118, 2783, DOI: https://dx.doi.org/10.1002/jgra.50325

Munakata, K., Yasue, S., Kato, C., et al., 2006, Advances in Geosciences: Volume 2: Solar Terrestrial (ST) (Singapore: World Scientific), 115

Petukhova, A. S., Petukhov, I. S., Petukhov, S. I., 2019, JGRA, 124, 19, DOI: https://dx.doi.org/10.1029/2018JA025964 
Potgieter, M. S., 2013, Living Reviews in Solar Physics 10.1:3, DOI: https://dx.doi.org/10.12942//rsp-2013-3

Richardson, I., Cane, H., 2011, SoPh, 270, 609, DOI: https://dx.doi.org/10.1007/s11207-011-9774-x

Tortermpun, U., Ruffolo, D., Bieber, J., 2018, ApJL, 852, L26, DOl: https://dx.doi.org/10.3847/2041-8213/aaa407

Usoskin, I. G., Gil, A., Kovaltsov, G. A., Mishev, A. L., Mikhailov, V. V., 2017, JGRA, 122, 3875, DOI: https://dx.doi.org/10.1002/2016JA023819

Wibberenz, G., Le Roux, J., Potgieter, M., Bieber, J., 1998, SSRv, 83, 309, DOI: https://dx.doi.org/10.1023/A:1005083109827

Zhang, G., Burlaga, L., 1988, JGRA, 93, 2511, DOI: https://dx.doi.org/10.1029/JA093iA04p02511

\section{Questions and answers}

Ludwig Klein: How do you define "class of events"?

Answer: The class of events is defined by FDs associated with ICMEs that do not have an effective shock/sheath part, for which isotropy of the GCR at the start time of the MC passage can be reasonably assumed. Moreover, for such events the observed modulation on GCRs is mainly due to the interaction with the MC.

Hugh Hudson: Can models based on diffusive propagation do as well?

Answer: The scale of the analyzed structure suggests that diffusive propagation might play a minor role in this case. In general a diffusive propagation model is more suitable to investigate the particle propagation on larger scales.

David Ruffolo: Entry/exit of particles due to gradient \& curvature drifts was also considered by Krittinatham \& Ruffolo (2009).

Answer: Yes, indeed we took into account the paper by Krittinatham \& Ruffolo (2009) in our study. 